\title{
Influencing Factors of Athletes' Injury Rehabilitation from the Perspective of Internal Environment
}

\author{
Xiang Huang ${ }^{1}$ and Xiaoping Wang $\mathbb{D}^{2}$ \\ ${ }^{1}$ School of Physical Education and Health, Yulin Normal University, Yulin 537000, China \\ ${ }^{2}$ School of Biology and Pharmacy, Yulin Normal University, Yulin 537000, China \\ Correspondence should be addressed to Xiaoping Wang; 201410730212@stu.shmtu.edu.cn
}

Received 23 July 2021; Revised 8 August 2021; Accepted 9 August 2021; Published 2 September 2021

Academic Editor: Yuanpeng Zhang

Copyright (c) 2021 Xiang Huang and Xiaoping Wang. This is an open access article distributed under the Creative Commons Attribution License, which permits unrestricted use, distribution, and reproduction in any medium, provided the original work is properly cited.

\begin{abstract}
Athlete's injury recovery is related to the athlete's personal value. A scientific and effective rehabilitation program will help athletes overcome their illnesses and return to the game as soon as possible. Based on the literature review and the internal environment perspective, this paper constructs a model of factors affecting athletes' injury rehabilitation. Through the empirical analysis of 129 questionnaires, we have verified the research hypothesis of each factor. The research results show that psychological adjustment, rehabilitation learning, and video reflection have a significant positive impact on athletes' injury rehabilitation, while imagery has no significant impact. This research provides a reference plan for athletes to adopt effective injury rehabilitation training methods. At the same time, we have also enriched the research literature on athletes' injury rehabilitation solutions.
\end{abstract}

\section{Introduction}

A healthy body is a key element for athletes to maintain their competitive advantage [1]. However, once an athlete is injured and fails to recover smoothly, it may affect the athlete's entire career [2]. Basketball player McGrady and hurdler Liu Xiang are typical examples. These athletes were plagued by injuries, and due to the poor recovery of the injury in the later period, the sports career came to an abrupt end. In addition, short-term injuries and poor recovery may also reduce the commercial value of athletes. For example, due to major athletes' injuries, NBA players may not be able to obtain a "big contract" during the health period when trading.

Sports injury rehabilitation [3] refers to comprehensive measures taken against dysfunction caused by athletes' injuries (e.g., bones, joints, muscles, and ligaments) [4]. The purpose is to improve and enhance the athletic function of athletes, so that patients can return to society [4]. At present, the main rehabilitation measures for athletes' injuries include physical therapy (e.g., exercise therapy and physical therapy), occupational therapy, and the application of orthotics and assistive devices [5]. Due to the huge consequences of athletes' injuries, various countries and teams are paying more and more attention to the rehabilitation and prevention of injuries.

Many scholars have carried out relevant research on specific measures for athletes' injury rehabilitation. For example, Hare et al. [1] and Sordoni et al. [4] studied the influence of intention on athletes' injury rehabilitation. Forsdyke et al. [2] analyzed the influence of psychological factors on athletes' injury rehabilitation through literature review; King et al. [6] put forward that authorization, participation, providing feedback, and transparency are four important habits to improve the effectiveness of athletes' injury rehabilitation. It can be seen that there are various measures for athletes' injury rehabilitation, and this is a process that is affected by both internal and external environments. In order to enhance the accuracy of the research, we only discuss the question of athletes' injury rehabilitation from an internal perspective in this paper. This paper have answered the question of what factors affect 
the rehabilitation of athletes' injury from the internal perspective. The research data of this paper was collected from the questionnaire and is analyzed by the SmartPLS software. Our study has enriched the related literature on athletes' injury rehabilitation; in particular, based on the internal perspective, we examined the effects of psychological adjustment, imagery, rehabilitation learning, and learning from the video on athletes' injury rehabilitation. In addition, this paper provided a feasible reference plan for athletes' injury rehabilitation.

The other parts of this research are arranged as follows: The second part is a literature review; the third part designs the theoretical framework of the research and puts forward the research hypothesis; the fourth part is the research methods and empirical results; the fifth part is the conclusion.

\section{Literature Review}

2.1. Athletic Injuries. Athletic injuries, as a common exercise disease, refer to various injuries that occur during exercise [7]. Generally speaking, the location of sports injury is related to sports events and specific technical characteristics [8]. For example, the injured parts of basketball players are mostly feet, wrists, shoulders, and waist. This is because there are more technical movements such as jumping, running, reclining, shooting, and accelerating in basketball [9].

There are many reasons for athletes' injuries, but they can be roughly divided into five categories $[10,11]$ : (1) insufficient training level, (2) irregular exercises, (3) failure to do preexercise or insufficient preexercise, (4) unintentional injury of other athletes, and (5) improper organization of competition work. Typical symptoms of athletic injuries include pain, swelling, and limited movement [10]. Athletic injuries can be identified by $\mathrm{X}$-ray, magnetic resonance imaging, or CT $[8,11]$.

Once a sports injury occurs, it needs to be checked and treated in time [12]. Because athletes' injuries are more acute than chronic, improper or untimely treatment of acute injuries may miss the best opportunity for injury treatment on the one hand, and on the other hand, it may also lead to the conversion of acute injuries to chronic injuries [13]. Therefore, athletes' injuries need to be taken seriously by athletes, team staff, and sports delegations from all over the world.

2.2. Measures for Injury Rehabilitation. Sports injury rehabilitation refers to the treatment measures taken after injury [14], which is fundamentally different from injury prevention. Due to the different parts of the body injured by athletes, there are also differences in specific rehabilitation measures [15]. Due to research needs, this article mainly discusses universal rehabilitation measures and their influencing factors based on the internal environment perspective.

The internal environment perspective [16], in which specialized rehabilitation strategies focused on the athletes themselves are discussed. For example, Gledhill and Ivarsson [17] proposed that athletes' injuries can cause athletes to experience psychosocial stress, anxiety, and emotional dis- tress. Furthermore, their ability to make rational decisions about rehabilitation activities may also be affected. Therefore, psychosocial factors have an important influence on the rehabilitation of athletes [18]. Forsdyke [19] proposed that imagery has a positive effect on sports injury rehabilitation. Specifically, imagination can help improve the patient's injury experience during the rehabilitation process. In addition, deep learning [20-22] and vision-based technologies [23-25] are also widely used in the field of sports injury rehabilitation research.

The above rehabilitation measures for athletes' injuries are all based on the athlete's internal perspective. It can be seen that based on the perspective of the internal environment, scholars have provided a wealth of literature on sports injury rehabilitation. However, relatively speaking, what is the effect of these internal measures on the rehabilitation of athletes, and what is the relationship between them? These issues need to be discussed in depth.

\section{Theoretical Framework and Research Hypothesis}

3.1. Theoretical Framework. Based on the above literature analysis, this paper constructs a model of factors affecting athletes' injury rehabilitation based on the internal environment perspective. See Figure 1 for details. The theoretical model mainly includes 4 internal factors, namely, psychological adjustment, imagery, rehabilitation learning, and video reflection. The two factors of psychological adjustment and imagination mainly come from previous studies. Rehabilitation learning and learning from the video are proposed in this article. Regarding the specific role of the latter two factors, we will elaborate in the research hypothesis section.

\subsection{Research Hypothesis}

3.2.1. Psychological Adjustment. Psychosocial factors are increasingly recognized as important factors affecting the rehabilitation and treatment of athletes [26] because, in sports, psychological factors are as important as the physical ability and talent of athletes. Especially after athletes' injuries, athletes need to release the pressure through psychological adjustment, so as to maintain an optimistic and positive attitude [27]. The study of Forsdyke et al. [2] found that athletes' emotions have a clear relationship with rehabilitation effects, and injuries, athletes' fear, anxiety, and selfconfidence are related to rehabilitation results. The research of Gledhill and Ivarsson [17] also shows that athletes' injuries often cause athletes' psychological stress, anxiety, and emotional distress. If these psychological problems are not handled well, they may affect their recovery process. It can be seen that psychological adjustment has a greater impact on athletes' injury rehabilitation. Ahern and Lohr [26] believe that athletes have good psychological adjustment capabilities, which will help their recovery from later injuries. Based on the above analysis, we put forward the following hypotheses: 


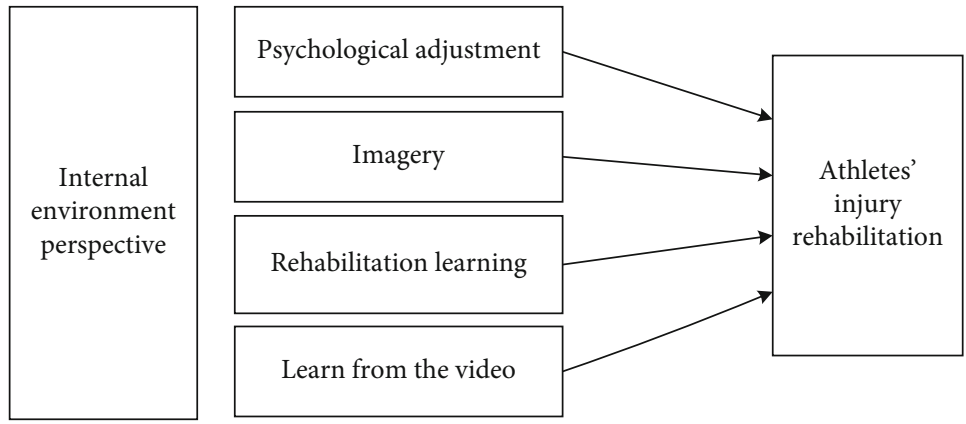

FIGURE 1: Research framework.

TABLE 1: Scale.

\begin{tabular}{llc}
\hline Latent variables & Observed variables & References \\
\hline Psychological & 1a: I think psychological adjustment is very important for injury recovery. & Gledhill and Ivarsson [17]; \\
adjustment (PA) & 1b: I think good psychological adjustment can help me overcome pain. & Ahern and Lohr [26] \\
& 1c: I think that recovery from injury cannot be separated from self-adjustment. & \\
\hline
\end{tabular}

2a: I often use imagery in the course of injury rehabilitation.

Imagery (I) 2b: I think the use of image therapy to treat pain can improve our satisfaction with recovery.

Hare et al. [1]; Law et al. [29]

2c: I think image therapy can be used for pain management.

3a: I often learn rehabilitation-related skills during the injury rehabilitation period.

Rehabilitation $\quad 3 b$ : I often learn about injury prevention during the injury recovery period.

learning $(\mathrm{RL}) \quad 3 \mathrm{c}$ : I often learn theories related to improving the professional level of sports

Jespersen [30]; Eastman and during the injury recovery period.

4a: I often watch my own sports videos during the injury recovery period to reflect and learn.

Learn from the video $4 \mathrm{~b}$ : During my injury rehabilitation period, I often watch sports videos of $(\mathrm{LftV})$ outstanding athletes in the same field to reflect and learn.

Wang and Parameswaran [32]

$4 \mathrm{c}$ : I think learning from the video will help me through the difficult rehabilitation period.

a: I think the process of injury recovery is affected by many factors.

Athletes' injury $\quad$ b: I think the key to good injury recovery lies in having a scientific coping style.

rehabilitation (AIR) c: I think the injury recovery period is not only a recovery process but also a good

Dhillon et al. [5] learning process.

H1: The stronger the athlete's psychological adjustment ability, the more helpful it is to recover from athletes' injuries.

3.2.2. Imagery. Imagery is widely used in practical treatment as a scientific rehabilitation method for athletes' injuries [4]. Green [28] studied the influence of imagery on sports injury rehabilitation earlier, and he demonstrated the application of imagery techniques in the chronological sequence of athletes' injuries. His research found that describing standards related to performance, athletes can use it to compare their progress in the recovery process [28]. The study of Hare et al. [1] shows that imagery ability has an impact on athletes' perception of injury response and imagination use function. In addition, a study by Law et al. [29] found that athletes use imagery for pain management, outdoor training, and competition environments. Athletes' use of image therapy to treat pain can increase their satisfaction with recovery. Accordingly, we propose the following research hypotheses:

$\mathrm{H} 2$ : Athletes use image therapy to help their recovery from athletes' injuries.

3.2.3. Rehabilitation Learning. Rehabilitation learning refers to learning skills related to rehabilitation, knowledge related to injury prevention, and theories related to improving the professional level of sports after an athlete's injury [30]. During the rehabilitation process, it is very important for athletes to maintain continuous learning. Athletes should shift from passive rehabilitation to active rehabilitation from injury. In this way, athletes are required to carry out necessary rehabilitation studies [31]. Learning skills related to rehabilitation can help athletes quickly adapt to their rehabilitation life; learning knowledge related to injury prevention 
TABLE 2: Descriptive statistical results of the survey.

\begin{tabular}{|c|c|c|}
\hline Basic information & Items & Rate \\
\hline \multirow{3}{*}{ Age } & $18-25$ & $68 \%$ \\
\hline & $26-30$ & $29 \%$ \\
\hline & $>30$ & $3 \%$ \\
\hline \multirow{2}{*}{ Gender } & Female & $41 \%$ \\
\hline & Male & $59 \%$ \\
\hline \multirow{2}{*}{ Whether they are recovering from injury } & Yes & $100 \%$ \\
\hline & No & $0 \%$ \\
\hline \multirow{4}{*}{ Sports field } & Track and field & $17 \%$ \\
\hline & Ball & $35 \%$ \\
\hline & Swimming & $8 \%$ \\
\hline & Other & $40 \%$ \\
\hline \multirow{3}{*}{ Education level } & Junior college or below & $6 \%$ \\
\hline & Bachelor's degree & $73 \%$ \\
\hline & Master degree or above & $21 \%$ \\
\hline
\end{tabular}

can help athletes avoid reinjury in the later period and reduce the probability of later injury of athletes; it assists players in entering the training environment in advance and preparing for their comeback after recovery by learning theories connected to enhancing the professional level of sports. Based on the above analysis, we propose the following hypotheses:

H3: Athletes use rehabilitation learning therapy to help their recovery from athletes' injuries.

3.2.4. Video Reflection. Video reflection refers to athletes who reflect and learn by watching their own sports videos and sports videos of outstanding athletes in the same field during the recovery from injury. The methods of analyzing and learning videos can be seen in many sports events [32]. For example, before a basketball game, many NBA teams will broadcast the opponent's game video to analyze the characteristics of the opponent's players and team tactics. Athletes watch their own sports videos during their rehabilitation. On the one hand, they can find improper actions that can easily lead to injuries from the videos. On the other hand, they can help maintain a good competitive state. And learn the sports videos of outstanding athletes in the same field, you can compare and analyze your own technical actions and then summarize the subsequent technical actions that can be improved. Accordingly, we propose the following research hypotheses:

H4: Athletes use video-retrospective therapy to help their recovery from athletes' injuries.

\section{Research Methods and Results}

4.1. Survey Design. In order to identify the factors affecting athletes' injury rehabilitation based on the internal environment perspective, we conducted a questionnaire design based on research hypotheses. The questionnaire mainly includes two parts. The first part is the basic information of the surveyed person (injured athletes who are in the
TABLE 3: Results of reliability and validity measurement.

\begin{tabular}{lccc}
\hline Construct & CA & CR & AVE \\
\hline PA & 0.758 & 0.823 & 0.642 \\
I & 0.713 & 0.785 & 0.733 \\
RL & 0.765 & 0.812 & 0.657 \\
LftV & 0.834 & 0.877 & 0.769 \\
AIR & 0.791 & 0.836 & 0.699 \\
\hline
\end{tabular}

recovery stage), including age, gender, education level, sports engaged in, and whether they are in the injury recovery stage. Meanwhile, the basic information in the first part is the control variables of this study. The second part is the four research variables (mental adjustment, imagery, rehabilitation learning, and video reflection) and one target variable (the athlete's injury rehabilitation) proposed in the previous article. In the specific operation, we use the 5point Likert scale to quantify the observed variables of each latent variable $[33,34]$. On the basis of referring to previous studies, we developed the scale shown in Table 1.

4.2. Data Collection. In order to ensure the validity of the questionnaire, we invited 4 athletes in the rehabilitation period to conduct a pretest of the questionnaire before the formal survey. At the same time, we have corrected some ambiguous items based on their feedback. Our questionnaire is mainly conducted online. The investigation will start in May 2021 and will be completed in July 2021. A total of 165 questionnaires were returned in this survey, of which 129 were valid questionnaires. The effective rate of questionnaires returned was $78.2 \%$. The survey was conducted anonymously, and all the information of the respondents was only used for scientific research. The descriptive statistical results of this survey are shown in Table 2.

4.3. Data Analysis. This paper uses the SmartPLS software and PLS-SEM data modeling [35] to conduct empirical 


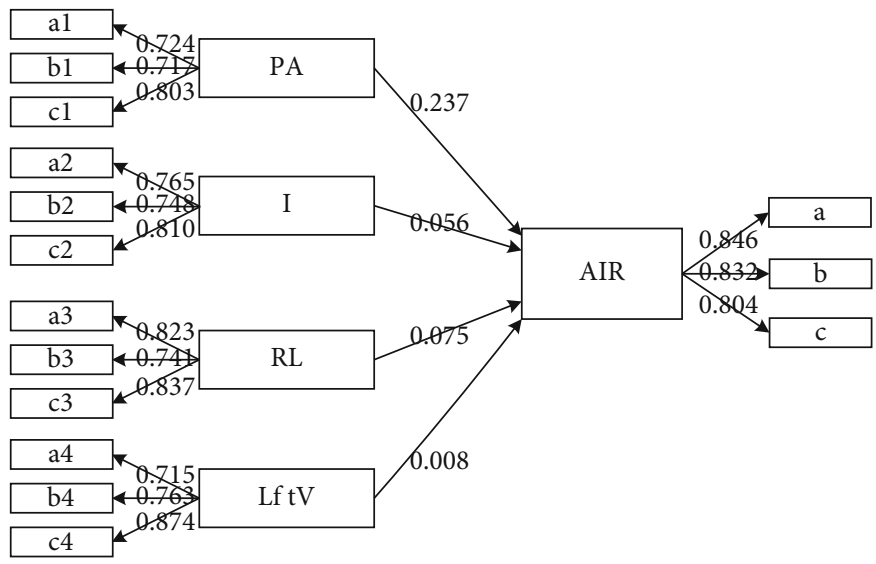

FIGURE 2: Model results.

research on the factors affecting athletes' injury rehabilitation. This research method has a very wide range of applications in the analysis of influencing factors. For example, Thaker et al. [36] used the SmartPLS software to study the factors that affect social media advertising. Silaparasetti et al. [37] used the SmartPIS software to analyze the impact of occupational health and safety on workers' behavior. It can be seen that this method is suitable for this study.

4.3.1. Reliability and Validity of Measurement. This paper analyzes the reliability and validity of the research scale and model. The results (see Table 3 ) show that the relevant indicators (Cronbach's alpha (CA), combined reliability $(\mathrm{CR})$, and average extraction variance (AVE)) have reached the corresponding threshold [38]. In addition, the external load value of each latent variable is greater than 0.7 (see Figure 2 for details). It can be determined that the scale and model of this study have good reliability and validity [38]. At the same time, it can be seen from the data analysis results in Table 4 that there is no multicollinearity among the latent variables [39]. The value of $R^{2}$ in the model is greater than 0.5 , indicating that the model constructed in this study has good explanatory power [39]. In addition, the value of the model fit in this paper is 0.035 , less than 0.06 , indicating that the model constructed has good adaptability [38].

4.3.2. Hypothesis Testing. This paper uses the SmartPLS software to verify the research hypothesis. The results are shown in Table 5. The research results show that hypotheses H1, $\mathrm{H} 3$, and $\mathrm{H} 4$ passed the hypothesis verification ( $P$ value is significant), while $\mathrm{H} 2$ did not pass the hypothesis verification ( $P$ value is not significant) [39]. From this, we can see that psychological adjustment, rehabilitation learning, and learn from the video have a significant positive impact on athletes' injury rehabilitation, while imagery has no significant impact. By comparison, we found that psychological adjustment, rehabilitation learning, and learn from the video support the majority of previous conclusions. However, our results suggest that imagery has no significant effect on athletes' injuries rehabilitation, which is contrary to the results of Hare et al. [1], Green [28], and Law et al. [29].
TABle 4: Average Variance Extracted (AVE) square root and factor correlation coefficient.

\begin{tabular}{lccccc}
\hline & $\mathrm{H}$ & $\mathrm{H} 1$ & $\mathrm{H} 2$ & $\mathrm{H} 3$ & $\mathrm{H} 4$ \\
\hline AIR & $\mathbf{0 . 9 4 7}$ & & & & \\
PA & 0.601 & $\mathbf{0 . 8 6 6}$ & & & \\
I & 0.426 & 0.576 & $\mathbf{0 . 7 8 7}$ & & \\
RL & 0.385 & 0.486 & 0.614 & $\mathbf{0 . 7 0 4}$ & \\
LftV & 0.233 & 0.358 & 0.332 & 0.431 & $\mathbf{0 . 6 9 2}$ \\
\hline
\end{tabular}

Diagonal elements are the square root of Average Variance Extracted (AVE).

TABLE 5: Results of hypothesis testing.

\begin{tabular}{lccccc}
\hline Hypothesis & Path & $\begin{array}{c}\text { Path } \\
\text { coefficient }\end{array}$ & $\begin{array}{c}t \\
\text { value }\end{array}$ & $\begin{array}{c}P \\
\text { value }\end{array}$ & $\begin{array}{c}\text { Hypothesis } \\
\text { supported? }\end{array}$ \\
\hline $\mathrm{H} 1$ & $\mathrm{PA} \rightarrow$ AIR & 0.237 & 3.560 & 0.000 & $\mathrm{Y}$ \\
$\mathrm{H} 2$ & $\mathrm{I} \rightarrow \mathrm{AIR}$ & 0.056 & 1.045 & 0.094 & $\mathrm{~N}$ \\
$\mathrm{H} 3$ & $\mathrm{RL} \rightarrow \mathrm{AIR}$ & 0.075 & 2.368 & 0.003 & $\mathrm{Y}$ \\
$\mathrm{H} 4$ & $\mathrm{LftV} \rightarrow \mathrm{AIR}$ & 0.008 & 1.981 & 0.037 & $\mathrm{Y}$ \\
\hline
\end{tabular}

\section{Conclusion}

Athlete's injury rehabilitation has always been a hot topic in academic circles. Scholars try to come up with solutions that help athletes recover from injuries from different perspectives. Based on the perspective of the internal environment, this paper constructs a model of factors that affect athletes' injury rehabilitation. In this article, we focus on the four factors of psychological adjustment, imagery, rehabilitation learning, and video reflection. Through questionnaire investigation and empirical analysis, we found that psychological adjustment, rehabilitation learning, and video reflection have a significant positive impact on athletes' injury rehabilitation, while imagery has no significant impact.

Our research provides a reference plan for athletes to adopt effective injury rehabilitation training methods. At the same time, we have also enriched the research literature on injury rehabilitation solutions. We emphasize that athletes need to maintain a good attitude during the injury 
repair period. For example, they can make psychological adjustments and release stress by talking with coaches, family members, and communicating. At the same time, the injury recovery period is also a period of learning and reflection. Athletes should seize this time without losing the opportunity to improve their theoretical level through knowledge learning and video analysis.

Our research has undeniable limitations. First, this article only discusses four factors that may affect athletes' injury rehabilitation based on the internal environment perspective. In the future, a variety of factors can be analyzed based on different perspectives. Second, the research sample in this article is 129 , so the research results have sample limitations. In the future, when conditions are mature, the sample size can be increased, and relevant factors can be explored. Finally, this article uses an online questionnaire collection method, so it is difficult to completely guarantee the authenticity of the surveyed information. In the future, offline or a combination of online and offline methods can be used to collect questionnaires.

\section{Data Availability}

The data used to support the findings of this study are included within the article.

\section{Conflicts of Interest}

All the authors do not have any possible conflicts of interest.

\section{References}

[1] R. Hare, L. Evans, and N. Callow, "Imagery use during rehabilitation from injury: a case study of an elite athlete," The Sport Psychologist, vol. 22, no. 4, pp. 405-422, 2008.

[2] D. Forsdyke, A. Smith, M. Jones, and A. Gledhill, "Psychosocial factors associated with outcomes of sports injury rehabilitation in competitive athletes: a mixed studies systematic review," British Journal of Sports Medicine, vol. 50, no. 9, pp. 537-544, 2016.

[3] X. Chen and G. Yuan, "Sports injury rehabilitation intervention algorithm based on visual analysis technology," Mobile Information Systems, vol. 2021, Article ID 9993677, 8 pages, 2021.

[4] C. Sordoni, C. Hall, and L. Forwell, "The use of imagery by athletes during injury rehabilitation," Journal of Sport Rehabilitation, vol. 9, no. 4, pp. 329-338, 2000.

[5] H. Dhillon, S. Dhilllon, and M. S. Dhillon, "Current concepts in sports injury rehabilitation," Indian journal of orthopaedics, vol. 51, no. 5, pp. 529-536, 2017.

[6] J. King, C. Roberts, S. Hard, and C. L. Ardern, "Want to improve return to sport outcomes following injury? Empower, engage, provide feedback and be transparent: 4 habits!," British Journal of Sports Medicine, vol. 53, no. 9, pp. 526-527, 2019.

[7] K. D. Peterson and L. C. Evans, "Decision support system for mitigating athletic injuries," International Journal of Computer Science in Sport, vol. 18, no. 1, pp. 45-63, 2019.

[8] M. Case, Athletic Injuries: Psychological Impacts and Athlete Preparedness to Return to Participation, Informa Healthcare, 2019.
[9] S. Abdollahi and R. Sheikhhoseini, "Sport-related injuries in Iranian basketball players: evidence from a retrospective epidemiologic study (2019-20)," The Physician and Sportsmedicine, 2021.

[10] T. Nakamura and H. Tsuchiya, "Association of hardiness and social support with posttraumatic growth following athletic injuries," International Journal of Sport and Health Science, vol. 18, pp. 28-38, 2020.

[11] A. N. Marshall, T. C. Valovich McLeod, and K. C. Lam, "Characteristics of injuries occurring during cross-country: a report from the athletic training practice-based research network," Journal of Athletic Training, vol. 55, no. 12, pp. 1230-1238, 2020.

[12] M. Stick-Mueller and M. Tunning, "The ICA Athletic Commission: an early effort to connect athletes to chiropractic," Chiropractic History, vol. 41, no. 1, 2021.

[13] R. Elwood, O. El-Hakeem, Y. Singh, H. Shoman, O. Weiss, and V. Khanduja, "Outcomes and rate of return to play in elite athletes following arthroscopic surgery of the hip," International Orthopaedics, pp. 1-11, 2021.

[14] G. Lei, "Application and research of nano-biomaterials in knee cruciate ligament rehabilitation after sports injury," International Journal of Nanotechnology, vol. 18, no. 1/2/3/4, pp. 82-96, 2021.

[15] S. Su, "Statistical calculation method and analysis of athletes' biorhythm state and sports injury," Mobile Information Systems, vol. 2021, 9 pages, 2021.

[16] A. Joseph, S. Gupta, Y. C. Wang, and K. Schoefer, "Corporate rebranding: an internal perspective," Journal of Business Research, vol. 130, pp. 709-723, 2021.

[17] A. Gledhill and A. Ivarsson, "Believe in your ability to create change: psychosocial factors influencing sports injury rehabilitation adherence," The Psychology of Sports Injury, pp. 93106, 2021.

[18] S. Pierce, The Importance of Psychosocial Considerations in the Injury Rehabilitation of Elite Athletes, A Senior Thesis Submitted in Partial Fulfillment of the Requirements for Graduation, 2021.

[19] D. Forsdyke, Seeing Is Believing: the Role of Imagery in Sports Injury Rehabilitation, Taylor \& Francis Group, 2021.

[20] W. Chu, Studies on the Effects of Wiring Density on Chip Package Interaction and Design Optimization with Machine Learning [Ph.D. thesis], 2021.

[21] J. Zhang, J. Sun, J. Wang, and X. G. Yue, "Visual object tracking based on residual network and cascaded correlation filters," Journal of Ambient Intelligence and Humanized Computing, vol. 30, pp. 1-4, 2020.

[22] Y. Gu, A. Chen, X. Zhang, C. Fan, K. Li, and J. Shen, "Deep learning based cell classification in imaging flow cytometer," ASP Transactions on Pattern Recognition and Intelligent Systems., vol. 1, no. 2, pp. 18-27, 2021.

[23] S. Qi, X. Ning, G. Yang et al., "Review of multi-view 3D object recognition methods based on deep learning," Displays, vol. 14, article 102053, 2021.

[24] M. Gao, W. Cai, and R. Liu, "AGTH-net: attention-based graph convolution-guided third-order hourglass network for sports video classification," Journal of Healthcare Engineering., vol. 2021, article 8517161, 10 pages, 2021.

[25] C. Yan, G. Pang, X. Bai et al., "Beyond triplet loss: person reidentification with fine-grained difference-aware pairwise loss," IEEE Transactions on Multimedia., vol. 31, 2021. 
[26] D. K. Ahern and B. A. Lohr, "Psychosocial factors in sports injury rehabilitation," Clinics in Sports Medicine, vol. 16, no. 4, pp. 755-768, 1997.

[27] L. Podlog, J. Heil, and S. Schulte, "Psychosocial factors in sports injury rehabilitation and return to play," Physical Medicine and Rehabilitation Clinics, vol. 25, no. 4, pp. 915-930, 2014.

[28] L. B. Green, "The use of imagery in the rehabilitation of injured athletes," The Sport Psychologist, vol. 6, no. 4, pp. 416-428, 1992.

[29] B. Law, M. Driediger, C. Hall, and L. Forwell, "Imagery use, perceived pain, limb functioning and satisfaction in athletic injury rehabilitation," New Zealand Journal of Physiotherapy, vol. 34, no. 1, 2006.

[30] E. Jespersen, "Peers as resources for learning: a situated learning approach to adapted physical activity in rehabilitation," Adapted Physical Activity Quarterly, vol. 25, no. 3, pp. 208227, 2008.

[31] A. Eastman and D. G. Chang, "Return to learn: a review of cognitive rest versus rehabilitation after sports concussion," NeuroRehabilitation, vol. 37, no. 2, pp. 235-244, 2015.

[32] J. R. Wang and N. Parameswaran, "Survey of sports video analysis: research issues and applications," in Proceedings of the Pan-Sydney area workshop on Visual information processing, pp. 87-90, Sydney, 2004.

[33] I. E. Allen and C. A. Seaman, "Likert scales and data analyses," Quality Progress, vol. 40, no. 7, pp. 64-65, 2007.

[34] B. Hou, W. Huanfang, and X. Guojie, "Study on the influencing factors of crowdsourcing logistics under sharing economy," Management Review, vol. 31, no. 8, p. 219, 2019.

[35] C. Ringle, D. Da Silva, and D. Bido, "Structural equation modeling with the Smartpls," Brazilian Journal of Marketing, vol. 13, no. 2, 2014.

[36] H. M. T. Thaker, A. Khaliq, A. A. Mand, H. I. Hussain, M. A. B. M. T. Thaker, and A. B. A. Pitchay, "Exploring the drivers of social media marketing in Malaysian Islamic banks: an analysis via smart PLS approach," Journal of Islamic Marketing, 2020.

[37] V. Silaparasetti, G. V. R. Rao, and F. R. Khan, "Structural equation modeling analysis using smart pls to assess the Occupational Health and Safety (OHS) factors on workers' behavior. Structural equation modeling analysis using smart PLS to assess the Occupational Health and Safety (OHS) factors on workers' behavior (July 17, 2017)," Humanities \& Social Science Reviews, pp. 2395-7654, 2017.

[38] P. U. Gio and E. Rosmaini, Belajar Olah Data dengan SPSS, Minitab, R, Microsoft Excel, EViews, LISREL, AMOS, dan SmartPLS, Universitas Sumatera Utara, Medan, Indonesia, 2016.

[39] K. K. K. Wong, "Partial least squares structural equation modeling (PLS-SEM) techniques using SmartPLS," Marketing Bulletin, vol. 24, no. 1, pp. 1-32, 2013. 